Relations industrielles

Industrial Relations

\title{
Unemployment in History, par John A. Garraty, Toronto, Fitzhenry and Whitheside Limited, 1979, 272 pp.
}

\section{André Roy}

Volume 35, numéro 1, 1980

URI : https://id.erudit.org/iderudit/029046ar

DOI : https://doi.org/10.7202/029046ar

Aller au sommaire du numéro

Éditeur(s)

Département des relations industrielles de l'Université Laval

ISSN

0034-379X (imprimé)

1703-8138 (numérique)

Découvrir la revue

Citer ce compte rendu

Roy, A. (1980). Compte rendu de [Unemployment in History, par John A. Garraty, Toronto, Fitzhenry and Whitheside Limited, 1979, 272 pp.] Relations

industrielles / Industrial Relations, 35(1), 162-165.

https://doi.org/10.7202/029046ar

Tous droits réservés (C) Département des relations industrielles de l'Université Laval, 1980
Ce document est protégé par la loi sur le droit d'auteur. L’utilisation des services d'Érudit (y compris la reproduction) est assujettie à sa politique d'utilisation que vous pouvez consulter en ligne.

https://apropos.erudit.org/fr/usagers/politique-dutilisation/ 
Tout autre est l'attitude des syndicats catholiques (et des Chevaliers du Travail qui les ont précédés dans le temps) face au libéralisme économique et aux valeurs nationales et au religieuses. Leur modèle d'action syndicale reste selon l'auteur marqué des rapports de l'âge préindustriel. Ils ne perçoivent pas les rapports employés/employeurs en terme d'opposition. Les syndicats catholiques vont même viser à un état d'harmonie entre patrons et ouvriers dans le cadre de la corporation, forme qui reste cependant assez indéterminée. Communauté des intérêts et complémentarité sont exaltés dans cette perspective.

L'auteur montre que les syndicats catholiques ont perdu dans leur pratique quotidienne beaucoup de l'idéalisme qui avait présidé à leur fondation. Ils se rallient à la négociation collective, délaissent l'arbitrage obligatoire pour la grève et en viennent à préconiser l'atelier syndical fermé. À la fin de la période étudiée, l'auteur constate un écart entre discours et pratique syndicale dans la CTCC, la pratique étant assez proche de celle des internationaux. L'idéologie des Chevaliers du Travail puis celle de la CTCC confinent, suivant l'auteur, pour une grande part à l'utopie. D'où leur échec relatif et ce jugement final: «Les sociétés industrialisées de type libéral laissent peu d'autres choix aux travailleurs que de concevoir leurs relations avec l'employeur sous le signe d'un rapport de forces. Le modèle corporatiste méconnaissait cette règle; il relevait d'une idéologie en désaccord avec une société industrialisée.»

En somme, cette étude fouillée dans l'analyse et vigoureuse dans la synthèse éclaire beaucoup l'aventure du syndicalisme national et catholique au Québec. Elle malmène aussi fort la légende de «syndicats jeunes prêts à tous les compromis avec les employeurs» propagée selon l'auteur par leurs adversaires internationaux.

Pierre SAVARD

Département d'histoire, Université d'Ottawa
Unemployment in History, par John A. Garraty, Toronto, Fitzhenry and Whitheside Limited, 1979, $273 \mathrm{pp}$.

Même si le chômage est au coeur des préoccupations de l'homme contemporain, il n'a peut-être jamais fait le sujet d'une analyse historique approfondie. Cependant, comme il le souligne en préface, l'auteur n'a pas voulu présenter une histoire du chômage en recherchant les raisons pour lesquelles il y a eu chômage à différentes époques ni en tentant de déterminer le nombre de personnes qu'il a touchées, mais plutôt il vise de mettre en lumière la perception qu'ont eue les hommes à travers l'histoire de «la condition d'être sans travail" et comment l'on a compris et évalué l'idée de chômage, même avant que le terme lui-même ne fut inventé.

L'ouvrage comprend treize chapitres qui, après un premier, où l'auteur s'efforce de cerner la notion moderne de chômage, décrivent, à partir de la période historique jusqu'à l'époque actuelle, les réactions des penseurs, des hommes politiques puis, avec le temps, des sociologues et des économistes devant la réalité de l'homme sans travail.

$\mathrm{Au}$ début de la période historique, le chômage n'était pas un problème social pour la bonne raison que la plupart des travailleurs étaient esclaves. Mais il est arrivé que les travailleurs libres en aient fait l'expérience. $\mathrm{Ne}$ se pourrait-il pas aussi que la construction des pyramides ou encore du temple de Jérusalem aient été imaginée comme de gigantesques projets de travaux publics pour occuper une main-d'oeuvre oisive ou que, avec la fin de ces entreprises, la concentration des ouvriers dans un même endroit ait provoqué une crise de chômage frictionnel? Un fait est certain: dans l'antiquité, En Egypte, en Grèce ou à Rome, il y avait chômage, sous-emploi, mendicité. Le panem et circences de la Rome impériale n'en est-il pas la preuve?

Qu'en fut-il au moyen âge? Le chômage n'y occupe pas une place importante, parce qu'il n'y avait à peu près pas de vie urbaine. Les méthodes de production étaient simples et l'agriculture vivière était l'occupation prin- 
cipale de la population. Puis, à ne pas oublier, l'auteur le signale au passage, le peuple au moyen-âge avait le sentiment profond d'un idéal d'interdépendance de tous les membres de la société. Ce n'est pas dire que la pauvreté n'existait pas, mais il y avait une volonté profonde d'aider les déshérités: d'où la mise sur pied de multiples oeuvres de bienfaisance.

Cependant, à mesure que la société s'urbanise, le nombre des pauvres s'accroit et on en vient à ranger sous cette dénomination tous ceux qui ne disposaient que de leur seule force de travail pour vivre sans distinguer entre les victimes du chômage et les autres laissés pour compte.

Au XVI et au XVIIe siècles, la situation a changé. L'Europe fait face à un accroissement du taux des naissances dont les effets immédiats sont d'augmenter le chômage, même si la découverte et la colonisation du continent américain suscitent la création d'emplois et appellent à des tâches nouvelles.

D'ailleurs, la situation varie d'un pays à l'autre. En Angleterre, par exemple, en 1618, Jacques I ordonne que la Virginie soit colonisée par des jeunes gens à la recherche de travail et un des premiers économistes, Josiah Child, écrit «Si nous avons en Angleterre de l'emploi pour cent personnes et que nous en ayons engendré et élevé cent cinquante, je dis que cinquante doivent partir, ou crever de faim ou être pendues».

Un seigneur français, La Popellinière, opine que la France peut exporter sans inconvénients le cinquième de sa population; mais en ce pays, l'émigration forcée soulevait de fortes oppositions, ce qui expliquerait peutêtre que, la situation étant moins grave en France qu'en Angleterre, les colonies anglaises se soient peuplées beaucoup plus rapidement que les colonies françaises. D'ailleurs, en ce dernier pays, Antoine de Montchrétien, l'inventeur de l'économie politique, estime que «les travailleurs sont les pieds de l'État, qu'ils soutiennent et portent tout le corps, qu'ils permettent à la noblesse d'exister et aux villes d'être alimentées».
Aussi, le chômage apparaît-il alors, moins comme une tragédie personnelle qu'une calamité nationale.

Cette conception du travail tend donc à faire considérer les sans-travail comme des paresseux, des gens sans initiative et sans volonté, ce qui conduit à des expressions d'opinions comme celle-ci: il faut tenir les basses classes dans la pauvreté ou elles ne seront pas industrieuses. Et malgré leur humanisme, leur compassion pour les pauvres et leur foi dans les avantages de l'instruction, les philosophes de la deuxième moitié du XVIIIe siècle n'ont que mépris pour les classes populaires, ces canailles, ces imbéciles ou ces idiots.

Mais, au XIXe siècle, c'est la grande transition avec l'avènement de la Révolution industrielle. Cet événement majeur de l'histoire du monde occidental va avoir une influence marquée sur les attitudes à l'égard du chômage. En effet, la Révolution industrielle réduit l'importance relative du travail en attirant femmes et enfants sur les marchés de l'emploi; elle accélère la concentration de la population dans les villes; elle crée le régime des manufactures; elle accroît l'efficacité du travail qui devient de plus en plus monotone; elle augmente l'importance des capitaux et le pouvoir des capitalistes dans la société; elle favorise l'explosion des taux de natalité; elle provoque de grandes fluctuations dans l'activité économique.

Ces transitions amènent les penseurs à se pencher sur la situation nouvelle, mais des hommes comme Adam Smith, Thomas Malthus, David Ricardo, n'ont que bien peu de chose à dire sur le phénomène du chômage qu'ils considèrent incontrolable.

Cependant, à mesure que les années s'écoulent et que se répand à travers le monde occidental le "système industriel britannique», on se rend compte, malgré l'expansion de la richesse, que la pauvreté persiste, qu'il y a surplus de main-d'oeuvre et que, de plus en plus, la vie économique se déroule par alternances de périodes de prospérité et de crise. 
Ce sont là les faits que révèlent de nombreuses enquêtes sur la condition ouvrière comme celles de Villermé et de Frégier en France, des tentatives infructueuses d'établissements d'ateliers sociaux comme celui de Louis-Blanc. D'autre part, Marx lance sa célèbre théorie de l'armée de réserve des travailleurs en disponibilité. Ce sont aussi les études de Charles Booth en Grande-Bretagne et de Denis Poulot en France qui tentent de catégoriser les chômeurs selon leur capacité physique, leur propension à travailler. C'est ainsi que l'on en vient graduellement à découvrir le vrai visage du chômage, que sa nature se précise en tant que phénomène social et économique.

Mais à cette époque, le chômage apparaît encore à la plupart des observateurs comme un mal nécessaire du régime capitaliste. C'est pourquoi, au commencement du XXe siècle à la grande dépression de la décennie 1930, le chômage est considéré comme un problème d'industrie. Deux idées se dégagent cependant: il faut rechercher les moyens de restreindre et de prévenir le chômage et tenter de trouver les moyens d'aider ceux qui en sont victimes. C'est la période de la fondation des bourses du travail et des bureaux de placement, des campagnes de publicité en vue de diminuer le chômage saisonnier, etc. On commence aussi à songer à l'établissement de régimes d'assurance qui s'inspirent, par exemple, du système de Bond. L'idée en est toutefois critiquée. On craint que ces mesures amoindrissent le goût du travail de telle sorte qu'il faudra attendre l'expérience catastrophique de la grande dépression avant que les régimes d'assurancechômage tels qu'on les connaît aujourd'hui se généralisent. En d'autres termes, il faut apprendre et se résigner à vivre avec le chômage, d'autant plus que la période d'abondance d'après la première guerre mondiale projetait une prospérité sans fin.

Le réveil est brutal. En 1929, se déclenche la plus terrible crise économique que le monde occidental n'a jamais connue et, à l'exception de quelques régimes éphémères, elle va durer jusqu'à la deuxième guerre mon- diale, soit toute la décennie.

À cette période tragique de l'histoire économique et sociale, l'auteur consacre deux chapitres.

Le premier traite des réactions des hommes politiques et des économistes face au chômage et des effets de la dépression sur les masses. Les gouvernements ont été pris au dépourvu. Leur réaction est négative. Pour eux et ceux qui les conseillent, il faut s'habituer à vivre avec un pourcentage élevé de sans-travail. Par ailleurs, les statistiques sont loin d'être à point. Elles varient selon les pays et l'OIT se plaint de cette trop grande diversité. Comme on l'a fait par le passé, on recourt à la méthode des enquêtes monographiques. L'auteur en signale plusieurs, notamment celle faite auprès de la population de Marienthal en Autriche où une filature, seule entreprise de la localité, a dû fermer ses portes. En Angleterre, une enquête exhaustive est entreprise et les résultats en sont publiés en neuf volumes en 1931 et en 1935. Il $\mathrm{y}$ en eut également aux États-Unis, où les chercheurs soumettent les sans-travail à une avanlanche de tests, de questionnaires et d'entrevues par lesquelles on s'efforce de découvrir les conséquences psychologiques du chômage.

Qu'a-t-on trouvé au cours de ces études? On se rendit compte que la dépression n'eut pas de contrecoups dramatiques sur la santé de la population, même si l'alimentation était loin d'être satisfaisante. Faute de vêtements, des enfants étaient empêchés de fréquenter l'école. Psychologiquement, on s'aperçut que, à mesure que le chômage se prolongeait, les gens devenaient apathiques, résignés à leur sort. Même si l'universalité du chômage pouvait psychologiquement en aider un certain nombre à tenir le coup et à patienter, la majorité des sans-travail ressentaient leurs conditions comme une grande humiliation. Fait surprenant toutefois, la situation économique n'engendra pas beaucoup d'activité subversive, si ce n'est des assemblées de masse, des marches de la faim, etc. En effet, pour beaucoup de gens, le monde était au bord du chaos. 
La grande dépression, cependant, n'a pas été marquée qué par une élévation sans précédent des taux de chômage. Celui-ci était une conséquence plus qu'une cause. Au fond, c'est le déclin massif des valeurs monétaires et l'effondrement de la demande et de la production qui l'ont provoqué. C'est pourquoi il fallait, non seulement en chercher la guérison par des mesures d'aide aux sans-travail sous quelque forme que ce soit, mais trouver les moyens de remettre la machine économique en marche.

Au départ, la guerre y a pourvu. Mais il ne faut pas négliger l'apport des économistes et, de tous ceux-ci, un nom vient immédiatement à l'esprit: John Maynard Keynes dont le célèbre ouvrage General Theory of Employment, Interest, and Money a influencé et influence encore la politique économique de bien des nations occidentales. Pour Keynes, l'épargne n'était pas nécessairement une vertu. Ce qui importait, durant les dépressions, c'était de stimuler la consommation. Pour cela, il fallait entreprendre de vastes programmes de travaux publics, accroître le crédit, même au risque de provoquer l'inflation. Au point de vue pratique, cette phrase d'un autre économiste traduit bien la pensée de Keynes «Si quelqu'un emploie 100,000 personnes et leur paie des gages, elles dépensent ces gages et l'argent ainsi dépensé emploie d'autres hommes». Keynes visait donc à la réalisation du plein emploi. Il ne devait pas atteindre le but qu'il cherchait, parce qu'il y a bien d'autres formes de chômage que le chômage cyclique, mais sa théorie, une fois appliquée, a permis au monde occidental de connaître, au cours des trente dernières années une ère véritable de prospérité. Ce fut le triomphe de la société d'abondance et de la société de consommation.

Mais cette période touche-t-elle à sa fin avec l'apparition du nouveau phénomène connu sous le nom de stagflation? L'auteur y consacre les deux derniers chapitres de son ouvrage. Pour lui, c'est peut-être la fin de l'âge d'or. Si le chômage est une maladie des sociétés industrielles modernes, l'inflation est aussi une de ses caractéristiques, et les rapports entre l'un et l'autre sont intimes. Les vérités que Keynes a expliquées demeurent mais «la théorie générale» n'apparaît plus être tellement générale. Ne serait-elle pas un cas spécial qui n'est applicable que dans certaines conditions et à une certaine époque. $\mathrm{La}$ nôtre n'aurait-elle pas besoin d'un nouveau Keynes, mais ce sauveur ne semble pas encore être en vue.

Ce qui précède est un résumé bien pâle et fort incomplet d'un ouvrage fortement documenté. En entreprenant une synthèse de l'histoire de certains aspects du chômage à travers les âges, l'auteur s'engageait dans une entreprise difficile. Il n'a pas raté l'objectif et c'est ce qui rend la lecture de cet ouvrage à la fois captivante et utile: captivante parce qu'on peut y découvrir comment les hommes dans des siècles et des pays différents ont réagi devant la personne privée de travail ou incapable de travailler; utile, surtout dans les derniers chapitres, parce qu'elle oblige à réfléchir aux lendemains de l'époque actuelle où il faut faire face au cercle vicieux du chômage et de l'inflation juxtaposés.

André ROY

Québec

Grèves et lock-out au Québec - 1978, Rapport annuel, Québec, Services des études en relations et conditions de travail, Ministère du Travail et de la Main-d'oeuvre, juillet 1979, $69 \mathrm{pp}$.

Il s'agit du second rapport annuel sur le sujet publié par le MTMO; le premier ainsi qu'un document méthodologique préliminaire ont déjà été recensés dans les numéros 1 et 3 du volume 33 de Relations industrielles. Comme toute jeune publication, la présente connaît et connaîtra encore certaines modifications destinées à en améliorer la qualité et le volume d'information. Rappelons que "Grèves et lockout» vise à cerner au moyen de données statistiques un type de manifestation des conflits de travail, à savoir les arrêts 